\title{
PERFORMANCE EVALUATION OF THE NEW AMS SYSTEM AT ACCIUM BIOSCIENCES
}

\author{
Ugo Zoppi ${ }^{1} \bullet$ James Crye $\bullet$ Qi Song • Ali Arjomand \\ Accium BioSciences, Inc., 550 17th Avenue, Suite 550, Seattle, Washington 98122, USA.
}

\begin{abstract}
A new compact accelerator mass spectrometry (AMS) system dedicated to the measurement of radiocarbon has been commissioned at the Accium BioSciences headquarters in Seattle. The entire facility (including ancillary laboratories for the preparation of graphite targets) has been designed to handle samples with a wide range of ${ }^{14} \mathrm{C}$ concentrations. In this paper, we discuss the technical details of the new facility and present performance test results demonstrating state-of-theart capabilities. In particular, modern samples can be readily measured with $0.3 \%$ precision and accuracy, machine background levels are consistently in the low $10^{-16}\left({ }^{14} \mathrm{C} /{ }^{12} \mathrm{C}\right)$, and chemical background is approximately equivalent to a fraction of modern of 0.004. In addition, when 100-times-modern samples were processed, no increase in background was observed, either during sample processing or during AMS measurement. This corresponds to a dynamic range for ${ }^{14} \mathrm{C}$ analysis of 6 orders of magnitude.
\end{abstract}

\section{INTRODUCTION}

Biomedical applications are mistakenly considered to exclusively produce "hot" samples and requiring no more than $3-5 \%$ measurement precision. This is not always the case.

Blood plasma is the major compartment for transportation of drugs and metabolites in the body. Quantifying the concentration of drugs and metabolites over time provides detailed pharmacokinetic characterization of a novel drug in clinical trials. The concentration of a drug after a single dose may vary 4 orders of magnitude or more. Moreover, the concentration of minor drug metabolites may be 2 orders of magnitude or more below the concentration of the major metabolite. Quantifying this metabolite diversity within a dynamic system requires an AMS system operating at its full dynamic range. Furthermore, long-term projection of drug clearance based on short-term plasma concentration data requires highly reliable quantification of the drug at very low levels.

The recent release of the FDA's (U.S. Food and Drug Administration) guidance on conducting human drug studies at doses of $100 \mu \mathrm{g}$ or less (full text is available at http://www.fda.gov/cder/guidance/7086fnl.pdf) further establishes the need for a highly sensitive analytical platform with a wide dynamic range. A typical clinical study involves administration of a drug followed by repeated sampling of blood. The protocol may also involve collection of urine and feces. The chemical dose size varies depending on the type of study and may range from $<100 \mu \mathrm{g}$ to hundreds of milligrams. The radiochemical dose size is usually limited to $50-200 \mathrm{nCi}$ of radiocarbon.

Plasma samples may be measured "neat," in which case the expected results will be at or above modern levels. Urine samples may not have sufficient carbon to yield the desired amount of graphite. Also, high-performance liquid chromatography (HPLC) fractionation of samples produces individual fractions essentially devoid of carbon. In these cases, carrier carbon is added to produce sufficient graphite for AMS measurement. The expected AMS values in these samples may be well below modern levels since the carrier is nearly or entirely depleted of ${ }^{14} \mathrm{C}$. Measurement of ${ }^{14} \mathrm{C} /{ }^{12} \mathrm{C}$ in these samples requires the full extent of precision and sensitivity offered by a carbon-based AMS system.

The need for a reliable ${ }^{14} \mathrm{C}$ AMS system that can measure low ${ }^{14} \mathrm{C}$ concentrations prompted us to select the proven technology of a National Electrostatics Corporation (NEC) 0.5MV compact sys-

${ }^{1}$ Corresponding author. Email: uzoppi@acciumbio.com. 
tem (Sundquist et al. 1999; Suter et al. 2000). The single-stage, 250-kV acceleration unit (Schroeder et al. 2004) was also considered but later discarded as at the time there was no sufficient independent validation that it could provide the desired machine background level.

In the following, we discuss general features of the new AMS facility, including sample preparation laboratories, and present various performance test results.

\section{AMS FACILITY}

\section{General Layout}

The Accium BioSciences AMS facility is housed on the 5th floor of the James Tower Life Sciences Building located in downtown Seattle. The facility layout is custom-designed to facilitate unidirectional sample flow between different areas. In particular, to avoid any possible contamination by a "hot" substance, large biological samples believed to have a ${ }^{14} \mathrm{C}$ content equivalent to more than 100 times the modern ${ }^{14} \mathrm{C}$ concentration (i.e. $5 \mathrm{~mL}$ of plasma collected shortly after a ${ }^{14} \mathrm{C}$-labeled drug is administered), are prescreened in the Receiving Laboratory by liquid scintillation counting (LSC).

Consistent and stable environmental conditions in the accelerator hall are guaranteed by a 1000AC Schreiber Engineering (Cerritos, California, USA) water chiller. Coupled with a centralized, unidirectional-flow HVAC (heating, ventilation, air-conditioning) system, the chiller unit provides the necessary cooling power for all systems and stabilizes the room temperature for overnight unattended operation. With this set-up, we were able to limit temperature variation within a 24-hr period to $<1{ }^{\circ} \mathrm{C}$.

\section{AMS System}

The new AMS system was delivered in January 2006 and was formally commissioned in March 2006. The Accium Biosciences ${ }^{14} \mathrm{C}$ AMS system (see Figure 1) features a 40-sample Cs sputter cathode ion source, a National Electrostatics Corporation (NEC) Model 1.5SDH-1 Pelletron Accelerator, an analyzing system including a $90^{\circ}$ magnet and a multiple Faraday cup chamber for the measurement of ${ }^{12} \mathrm{C}$ and ${ }^{13} \mathrm{C}$ currents as well as a rare isotope beamline consisting of a $90^{\circ}$ electrostatic spherical analyzer and a final particle detector. This system employs the fast, $10-\mathrm{Hz}$ sequential-pulsing technique.

Presently, we have the capacity to prepare and analyze more than 10,000 samples per year. However, a 134-sample ion source has already been planned to provide dual-source capability (see Figure 1). This design will effectively eliminate downtime needed for servicing ion sources and loading new sets of samples. We anticipate an increased throughput up to 20,000 samples per year.

\section{Sample Preparation Laboratories}

The procedures for sample preparation have been modeled after Ognibene et al. (2003). Using this method, biological sample materials such as whole blood, plasma, urine, fecal homogenate, tissue homogenate, and HPLC fractions are transferred to individual baked quartz vials. In samples where the total carbon content is below $0.5-1.0 \mathrm{mg}$ (e.g. HPLC fractions or urine), a known amount of a suitable carbon carrier such as petroleum-based tributyrin is added. Water and volatile solvents are then removed from all samples in a vacuum centrifuge and the remaining residue is transferred to the Target Preparation Laboratory for conversion to graphite.

Firstly, each quartz vial containing the dried sample residue is sealed and combusted at $900{ }^{\circ} \mathrm{C}$ along with $200 \mathrm{mg} \mathrm{CuO}$ to form $\mathrm{CO}_{2}$. Samples are then attached to a disposable transfer system connected 


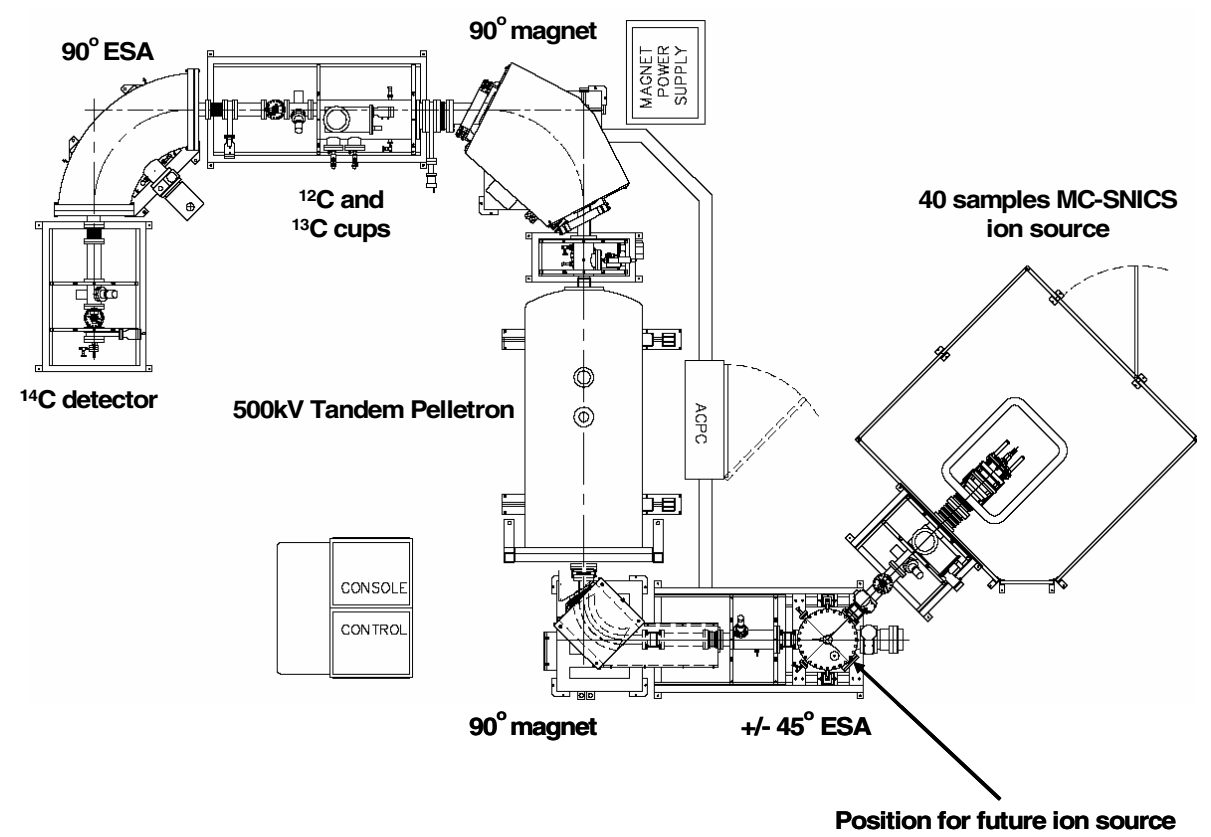

Figure 1 The Accium Biosciences ${ }^{14} \mathrm{C}$ AMS system. In particular, note that the ion source is placed at a $45^{\circ}$ angle to allow a possible future expansion with an additional ion source.

to a labeled septa-sealed vial. The vial contains $100 \mathrm{mg}$ of zinc, four 3-mm glass beads, and a smaller vial containing $\sim 5 \mathrm{mg}$ of iron powder. After evacuating the transfer system with a vacuum pump, the tip of the combustion tube is broken, allowing internal gases to flow into the septa-sealed vial, which is slightly submerged in liquid nitrogen. The liquid nitrogen causes gases such as $\mathrm{CO}_{2}$ and $\mathrm{H}_{2} \mathrm{O}$ to condense at the bottom of the vial while non-condensing gases are purged using the vacuum pump. To avoid cross-contamination, all parts that come into contact with the sample are disposable and are replaced with each use. Septa-sealed vials are then placed in a heat block for $5 \mathrm{hr}$ at $500{ }^{\circ} \mathrm{C}$. During this stage, carbon from $\mathrm{CO}_{2}$ is reduced to solid graphite, which adheres to the surface of the iron powder. The resulting iron-graphite mixture is pressed into individual cathodes and loaded onto the AMS sample wheel for measurement.

A typical sample batch contains 32 unknowns (biological samples), 4 certified standards to normalize all measurements, 2 machine blanks $\left({ }^{14} \mathrm{C}\right.$-free graphite of natural origin) to assess the sensitivity of the spectrometer, and 2 chemical blanks (samples prepared with a ${ }^{14} \mathrm{C}$-free substance) to characterize the extraneous carbon introduced during sample preparation. With the creation of each batch, every sample is assigned a unique tracking number representing the sample type, origin of the material, and the corresponding position on the AMS measurement wheel.

Biomedical studies generally require the separation and isolation of different chemical structures. For this reason, a HPLC system has been installed in the Processing Laboratory. Accium utilizes a Shimadzu Prominence HPLC system with a Shimadzu automated FRC-10A fraction collector. One common procedure is to collect HPLC eluent as a series of fractions in 30- to 60-s intervals. The fractions are collected directly in vials (ScreenMate tubes, Matrix Technology Corp.) placed within a 96-well format. When available, the identical non-radiolabeled reference chemical structure is premixed into the sample and co-injected onto the HPLC column to produce a detectable UV-VIS sig- 
nal. The peak on the UV chromatogram marks the retention time and elution fraction number of the corresponding ${ }^{14} \mathrm{C}$-chemical structure of interest, which is often present at levels well below the detection limit of the UV-VIS detector. The position of the peak in the UV chromatogram allows individual fractions to be pooled into a single sample for subsequent AMS measurement, providing the total ${ }^{14} \mathrm{C}$ signal from the peak of interest. Alternatively, all fractions are collected, individually processed and measured by AMS to produce a complete radio-chromatogram of all ${ }^{14} \mathrm{C}$-labeled structures separated by HPLC.

\section{PERFORMANCE EVALUATION}

The performance of the Accium AMS instrument and Graphite Preparation Laboratory was assessed in a series of validation tests carried out in accordance with the "Good Laboratory Practice for Nonclinical Laboratory Studies" (GLP) quality system (U.S. Food and Drug Administration, 21 CFR $58)$.

\section{Precision and Accuracy}

Precision and accuracy were firstly determined for the AMS machine alone by measuring large IAEA-C7 (Le Clercq et al. 1998), HOxI, and HOxII standards (Stuiver 1993) prepared at the Australian Nuclear Science and Technology Organization (ANSTO) (Fink et al. 2004; Qua et al. 2001). For each kind of standard, the graphite was pooled and thoroughly mixed before being loaded into different cathode holders. Three IAEA-C7 cathodes were each measured 3 times for 120 seconds and normalized against HOxI and HOxII standards and their results combined into a single mean value. The whole measurement sequence was repeated on 3 consecutive days. Results listed in Table 1 clearly show that for the first 2 days the fraction of modern $F$ and fractionation $\delta^{13} \mathrm{C}$ were determined with a precision and accuracy of better than $0.3 \%$ and $0.1 \%$, respectively. For the 3rd day, the fraction of modern $F$ was measured once again within $0.3 \%$ of the consensus value. However, evident cathode cratering limited us to $0.3-0.4 \%$ accuracy for $\delta^{13} \mathrm{C}$.

Table 1 Experimentally determined $F$ and $\delta^{13} \mathrm{C}$ values for IAEA-C7 standards (consensus values: $F=0.4954$ and $\delta^{13} \mathrm{C}=-14.5 \%$ ) measured on 3 consecutive days. By the end of the 3rd day, sample cratering limited the $\delta^{13} \mathrm{C}$ accuracy to $3 \%$.

\begin{tabular}{lll}
\hline Day & $F$ & $\delta^{13} \mathrm{C}$ \\
\hline 1 & $0.4949 \pm 0.0018$ & $-13.9 \%$ o \\
2 & $0.4944 \pm 0.0018$ & $-14.1 \%$ o \\
3 & $0.4934 \pm 0.0016$ & $-17.0 \%$ o \\
\hline
\end{tabular}

The performance of the Graphite Preparation Laboratory was assessed by producing and measuring a series of standard samples. The measurement sequence was very similar to the one discussed above. A set of IAEA-C6 and IAEA-C7 standards was prepared and measured against HOxII. Results displayed in Figure 2 demonstrate that an accuracy of $0.5 \%$ was achieved. The $0.2 \%$ difference in accuracy, compared to the values obtained with the graphite provided by ANSTO, is due to the different graphitization procedures.

\section{Background}

Machine background and chemical background were investigated with special emphasis on possible cross-contamination from "hot" samples. Two samples with fraction of modern of approximately 10 were prepared, each followed by a series of 5 chemical blanks. Cross-contamination in the Graphite 


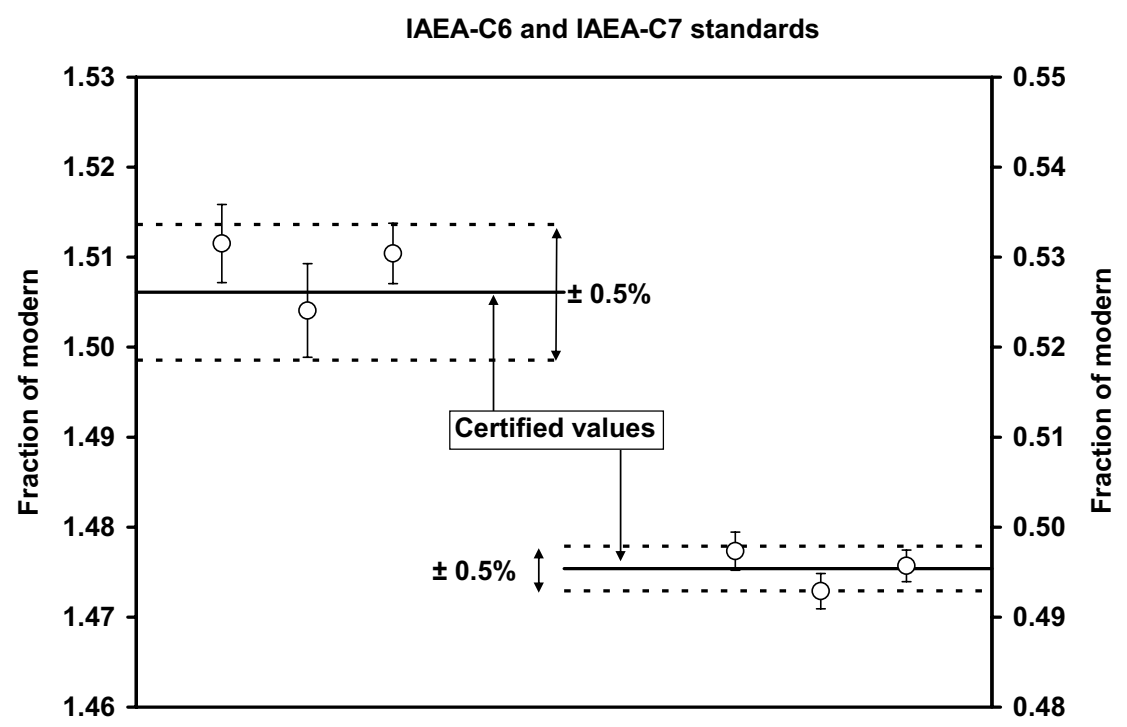

Figure 2 Comparison between measured and certified ${ }^{14} \mathrm{C}$ concentrations for IAEA-C6 and IAEA-C7 standards prepared at Accium.

Preparation Laboratory would be observed as an increase in ${ }^{14} \mathrm{C}$ concentration for the first chemical blank followed by a gradual decrease due to a "cleaning" process. During AMS measurement, after each "hot" sample a machine background sample was measured to investigate ion-source memory effects.

Our results (see Figure 3) were obtained by limiting the measurement time of "hot" samples to a maximum of $40,000{ }^{14} \mathrm{C}$ counts each time the cathode faced the Cs beam. This limiting procedure was instrumental in reducing source memory effects and allowed measurement of the machine background at $(2.7 \pm 0.5) \times 10^{-16}$, corresponding after normalization to a fraction of modern of $0.00023 \pm 0.00004$ and to a ${ }^{14} \mathrm{C}$ age of more than $67 \mathrm{kyr}$ BP. Values obtained for chemical blanks (equivalent to a ${ }^{14} \mathrm{C}$ age of more $45 \mathrm{kyr} \mathrm{BP}$ ) indicate that no cross-contamination took place during the reduction step.

To accurately characterize the extraneous carbon introduced during sample preparation, the procedure described by Hua et al. (2004) was used. Two series of samples of decreasing masses (IAEAC6 standard and tributyrin, a carrier commonly used for biomedical applications) were prepared and measured. The determined ${ }^{14} \mathrm{C}$ concentrations are clearly inversely proportional to the samples' mass (see Figure 4). This allowed us to calculate the mass $m_{c}$ and fraction of modern $F_{c}$ of the contaminant. Using the values from Figure 4, we estimate that the added contamination is equivalent to $m_{c}=10.7 \pm 0.6 \mu \mathrm{g} \mathrm{C}$ and $F_{c}=0.687 \pm 0.046$. The ${ }^{14} \mathrm{C}$ level is effectively the same as observed in other AMS facilities (see e.g. Hua et al. 2004). Although at Accium the calculated mass $m_{c}$ is higher by a factor 15 , it is clearly adequate to reliably measure samples containing at least $0.5 \mathrm{mg}$ carbon, equivalent to $10 \mu \mathrm{L}$ of plasma.

\section{Linearity and Reproducibility over the Full Dynamic Range}

The reproducibility of our chemical procedures and ${ }^{14} \mathrm{C}$ measurements was evaluated over the full dynamic range allowed by the AMS system by means of tributyrin samples spiked with different levels of ${ }^{14} \mathrm{C}$-enriched malonic acid to produce ${ }^{14} \mathrm{C}$ ratios with a fraction of modern ranging from 0.2 


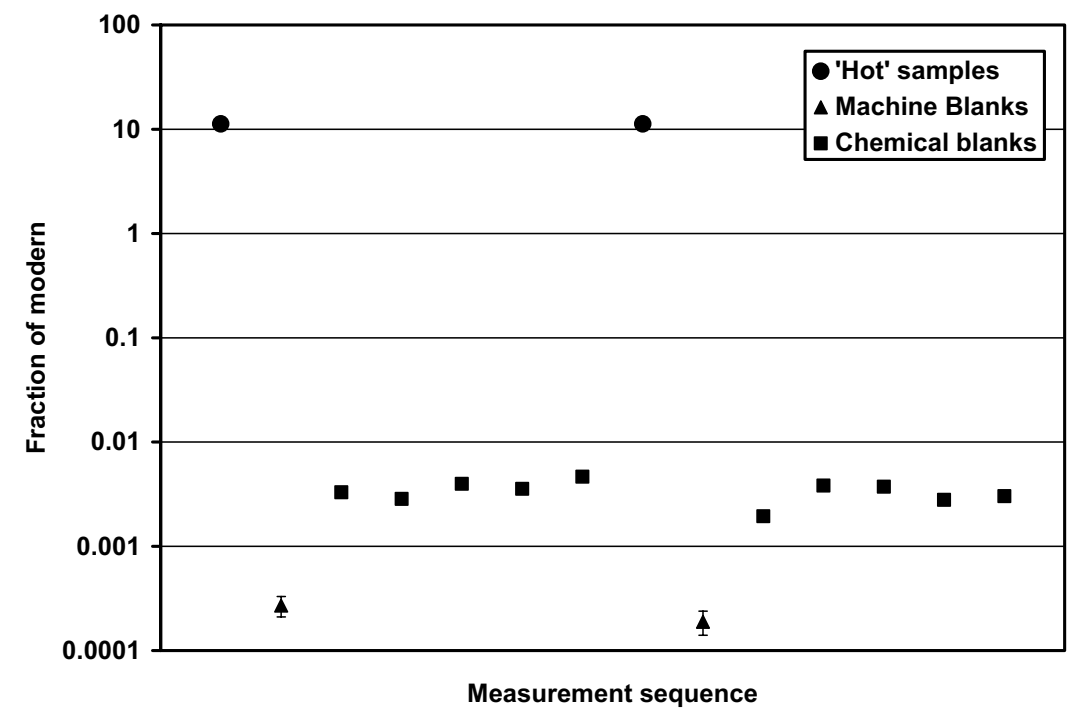

Figure 3 Measured ${ }^{14} \mathrm{C}$ concentrations for 2 "hot" samples followed by machine and chemical blanks demonstrate that there is no measurable cross-contamination. The measurement sequence (left to right) corresponds to the sequence in the sample processing laboratory. For further information, see text.

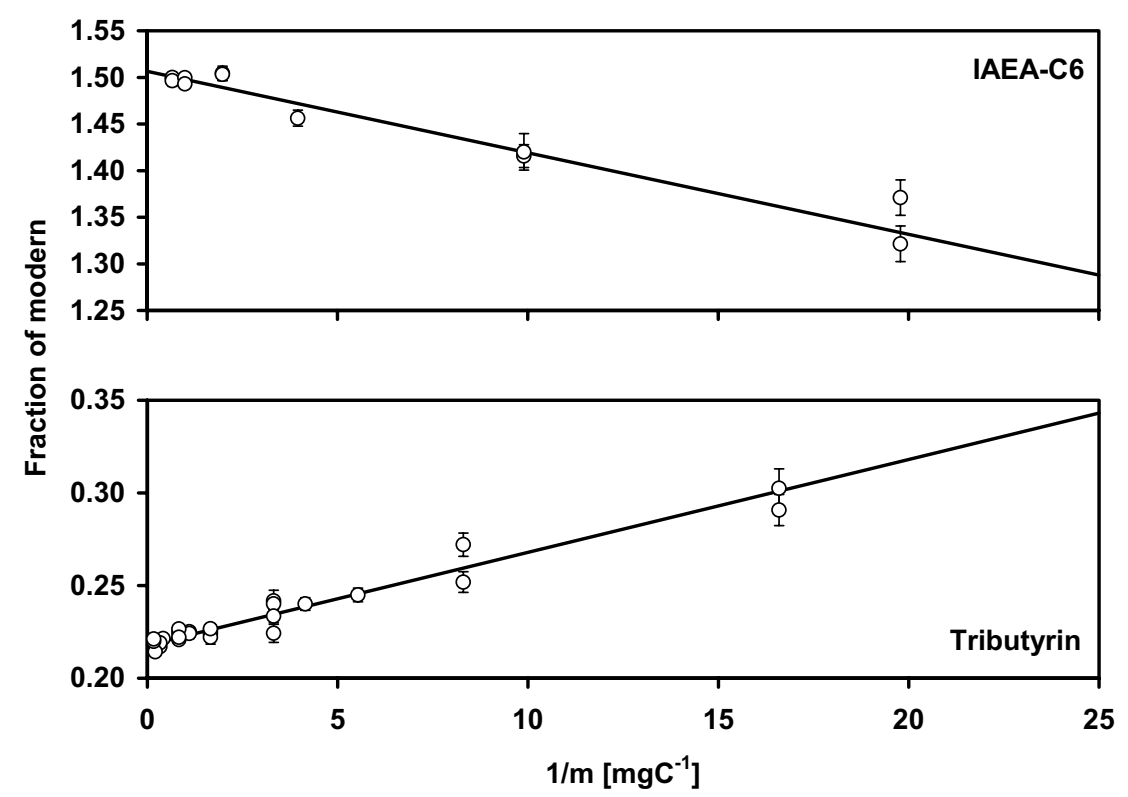

Figure 4 Measured ${ }^{14} \mathrm{C}$ concentrations for small mass samples used in characterizing extraneous carbon added during sample processing.

to 200. Each sample was prepared and measured in duplicate (Figure 5) and the average measured ${ }^{14} \mathrm{C}$ concentration was compared to the calculated concentration (Figure 6). Correlation coefficients of respectively $R^{2}=0.9995$ and $R^{2}=0.9987$ demonstrate extremely good accuracy and reproducibility for ${ }^{14} \mathrm{C}$ AMS measurements in biological samples across 6 orders of magnitude. 


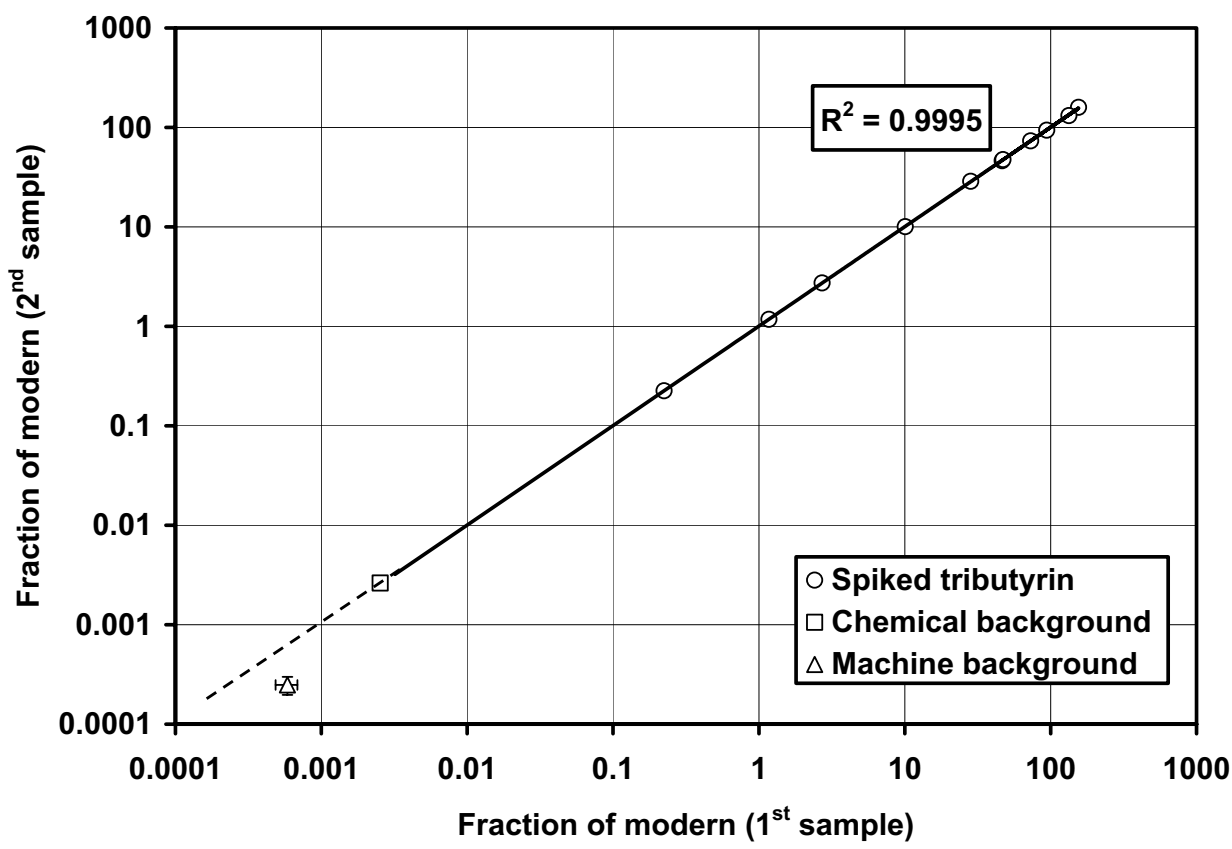

Figure 5 Comparison of measured ${ }^{14} \mathrm{C}$ concentrations for a series of duplicate samples demonstrates excellent reproducibility over an unprecedented 6 orders of magnitude. For further information, see text.

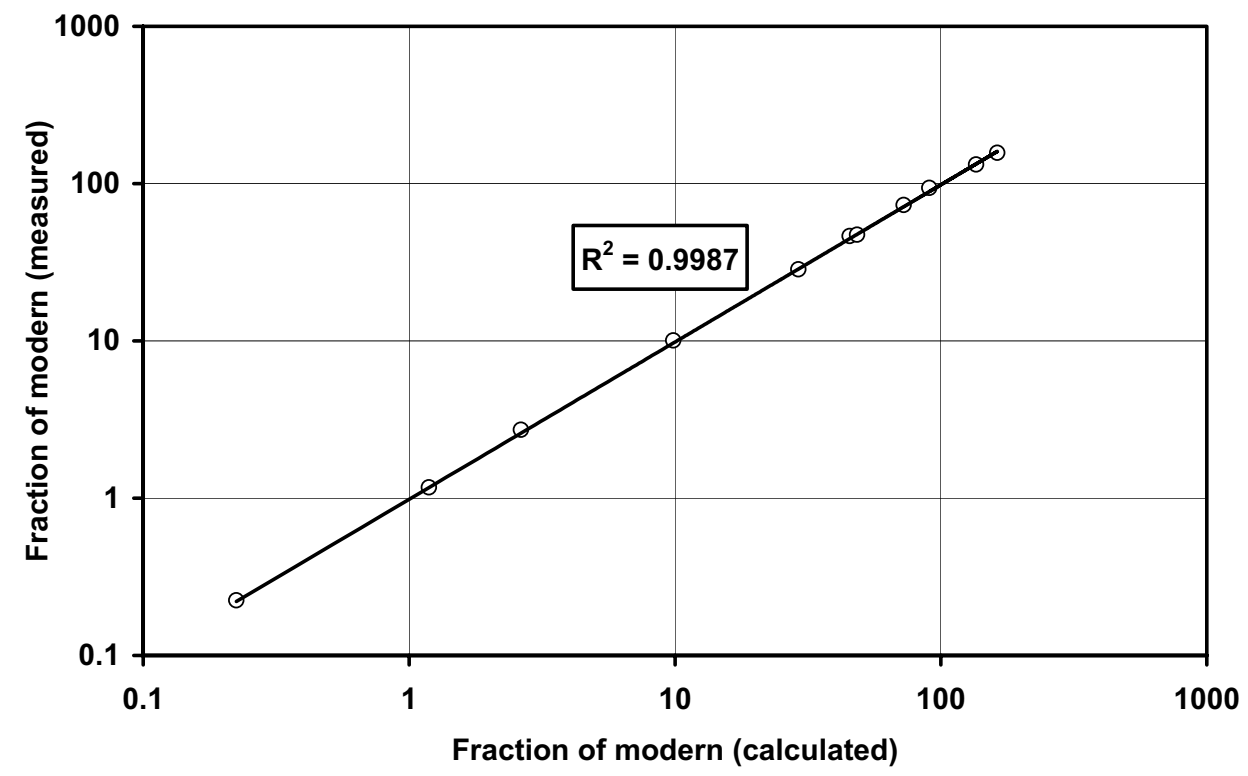

Figure 6 Linearity response curve for ${ }^{14} \mathrm{C}$ biological samples with ${ }^{14} \mathrm{C}$ concentrations between 0.1 and 150 modern. For further information, see text. 


\section{EXAMPLE OF BIOMEDICAL APPLICATION}

The diagnosis of Type II diabetes and the complications associated with the disease have risen to epidemic proportions in the last decade on a global scale. It is currently estimated that more than 150 million people suffer from Type II diabetes, with the characteristic hallmarks of insulin resistance in peripheral tissues, hyperglycemia, and pancreatic B-cell dysfunction. Although several marketed therapies are available, many have significant side effects or become ineffective for patients who receive these treatments. In addition, as disease progression occurs, the need for additional therapeutic intervention and supplemental insulin treatment is necessary. As such, considerable effort towards the development of therapeutic agents with novel mechanisms of action continues, with the hope to reduce the occurrence, progression, and complications of this debilitating disease.

One such approach is to obtain effective insulin sensitizing agents by targeting the insulin signaling pathway directly, and more specifically through Protein Tyrosine Phosphatase 1B (PTP1B). PTP1B is a ubiquitously expressed, non-receptor enzyme that negatively regulates insulin and leptin signaling in vivo. PTP1B knockout (PTP1B-/-) mice are healthy and show increased insulin sensitivity, improved glucose tolerance, and resistance to weight gain when fed a high-fat diet. In addition, studies from tissue-specific attenuation of PTP1B suggest a role for this enzyme in certain insulin sensitive tissues. Therefore, a small molecule reversible, competitive inhibitor of this well-validated target would provide a positive therapeutic benefit in treating Type II diabetes.

An early-stage highly-potent lead molecule (CEPTYR Inc. internal code CPT 377) from a chemical series designed to specifically inhibit PTP1B, was found to be efficacious in a mouse model of diabetes and obesity. Reductions in daily plasma glucose levels and positive effects in a standard glucose tolerance test were seen after 3-5 days of a once daily oral treatment with this compound. Traditional pharmacokinetic analysis revealed the oral bioavailability of the molecule to be low $(<6 \%)$, with a serum half-life of $1.5 \mathrm{hr}$. Based on the reported role of PTP1B in peripheral tissues of insulin sensitivity, an AMS study was designed to delineate if the positive efficacy results of this compound were due to residence time of the compound in specific tissues. This information would help establish the potential correlation between tissue exposure and effect.

In order to understand the variance between the efficacy and bioavailability of CPT 377 and to determine if the compound had enough residence time in insulin-sensitive tissues to provide an effect, an ultra-sensitive method for analyzing the distribution profile of low levels of compound was necessary. CPT 377 was easily labeled with ${ }^{14} \mathrm{C}$ in-house at a significant cost and time savings and was dosed to mice by oral gavage or intravenously, and samples collected at various time points through $24 \mathrm{hr}$. Distribution of the drug and its metabolites in plasma and various organs was determined by AMS using submilligram quantities of tissue and plasma (see Figure 7). The results demonstrated for the first time low drug distribution into target organs and provided evidence to shift the research effort to investigating alternate chemical modifications that improve potency, avoid rapid elimination, and target specific tissues of interest.

The study demonstrated the utility of AMS as an ultra-sensitive detection platform for quantifying drug kinetics and distribution in small animals. AMS analytical methods require no additional method development, including chromatographic or mass spectrometric optimization for specific chemical structures. This makes AMS even more suitable for early-phase drug development where analytical resources or methods may not be readily available, or where early preclinical characterization may help select the most suitable candidate within a series of similar compounds. As such, AMS-based protocols permit assessment of pharmacokinetic and ADME characteristics of new drugs earlier in development, with minimum bioanalytical development and tremendous sensitivity. 


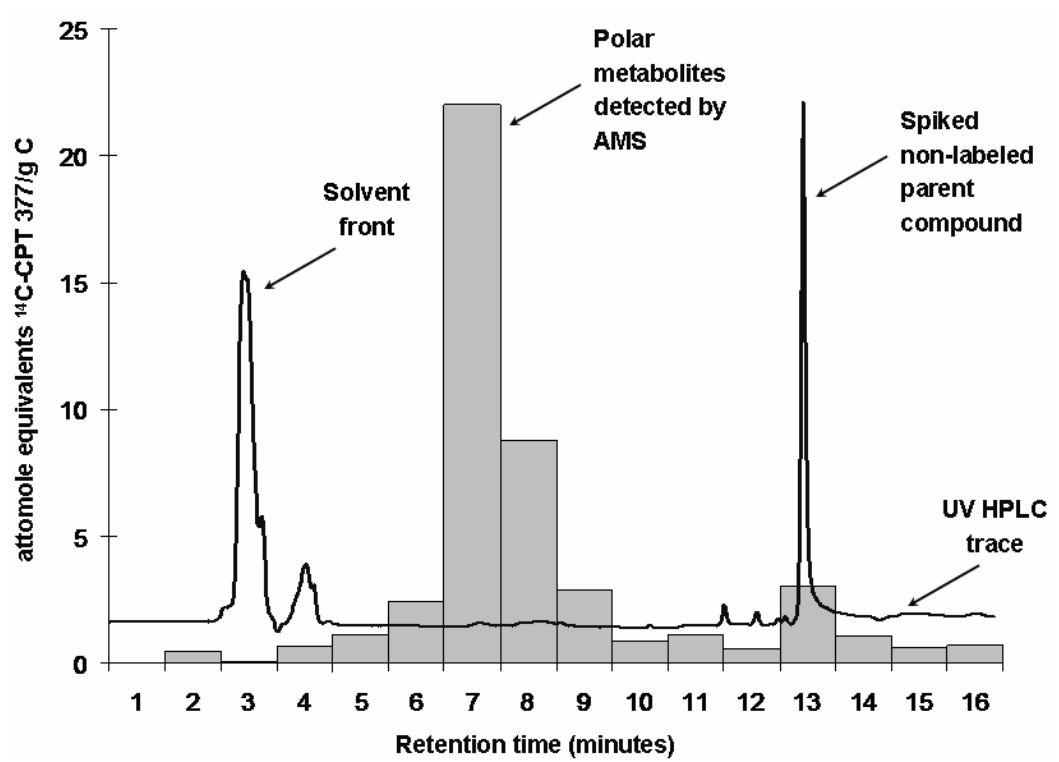

Figure 7 HPLC-AMS of mouse muscle sample 25 min after intravenous (IV) administration of lightly-labeled ${ }^{14} \mathrm{C}$-CPT 377 . An unlabeled parent compound was spiked into the sample prior to HPLC injection to mark the retention time of the intact molecule (retention time approximately $12.5 \mathrm{~min}$ ). HPLC fractions were collected at 1-min intervals and analyzed by AMS. The chart demonstrates metabolism of the majority of the administered dose into more polar forms appearing with a retention time of approximately $7-8 \mathrm{~min}$ in the HPLC trace. For the first time, tissue-level distribution and metabolite detection was demonstrated for this compound.

\section{CONCLUSION}

A new state-of-the-art AMS system has been commissioned by Accium BioSciences in Seattle. In particular, we demonstrated $0.3-0.5 \%$ precision and accuracy for modern samples processed in our laboratory. In addition, we also demonstrated the capability to handle samples with ${ }^{14} \mathrm{C}$ concentrations ranging over 6 orders of magnitude without any measurable cross-contamination.

\section{ACKNOWLEDGMENTS}

We gratefully acknowledge the Australian Nuclear Science and Technology Organization (ANSTO) for providing graphite targets for the first part of the performance assessment. We thank Andrew Clifford (University of California, Davis) and the Center for Accelerator Mass Spectrometry (CAMS) at Lawrence Livermore National Laboratory for suggestions prior to establishing our graphite preparation laboratory. We also thank Michele Luche (CEPTYR, Inc.) for her contributions during the CPT 377 investigation.

\section{REFERENCES}

Fink D, Hotchkis M, Hua Q, Jacobsen G, Smith AM, Zoppi U, Child D, Mifsud C, van der Gaast H, Williams A, Williams M. 2004. The ANTARES AMS facility at ANSTO. Nuclear Instruments and Methods in Physics Research B 223-224:109-15.

Hua Q, Jacobsen GE, Zoppi U, Lawson EM, Williams AA, Smith AM, McGann MJ. 2001. Progress in radiocarbon target preparation at the ANTARES AMS cen- tre. Radiocarbon 43(2A):275-82.

Hua Q, Zoppi U, Williams AA, Smith AM. 2004. Smallmass AMS radiocarbon analysis at ANTARES. $\mathrm{Nu}$ clear Instruments and Methods in Physics Research B 223-224:284-92.

Le Clercq M, van der Plicht J, Gröning M. 1998. New ${ }^{14} \mathrm{C}$ reference materials with activities of 15 and 50 pMC. Radiocarbon 40(1):295-7. 
Ognibene TJ, Bench G, Vogel JS, Peaslee GF, Murov S. 2003. A high-throughput method for the conversion of $\mathrm{CO}_{2}$ obtained from biochemical samples to graphite in septa-sealed vials for quantification of ${ }^{14} \mathrm{C}$ via accelerator mass spectrometry. Analytical Chemistry 75(9): 2192-6.

Schroeder JB, Hauser TM, Klody GM, Norton GA. 2004. Initial results with low energy single stage AMS. Radiocarbon 46(1):1-4.

Stuiver M. 1983. International agreements and the use of the new oxalic acid standards. Radiocarbon 25(2): 793-5.

Sundquist ML, Loger RL, Daniel RE, Kitchen RL, Kolonko JJ, Klody GM. 1999. Results of recent tests of NEC AMS systems. American Institute of Physics Proceedings 475:661-4.

Suter M, Jacob SWA, Synal H-A. 2000. Tandem AMS at sub-MeV energies-status and prospects. Nuclear Instruments and Methods in Physics Research B 172(14):144-51. 